ARTICLE

DOI: $10.1038 / s 41467-018-03471-x$

\title{
Photonic chip-based soliton frequency combs covering the biological imaging window
}

Maxim Karpov1, Martin H.P. Pfeiffer ${ }^{1}$, Junqiu Liuํ, Anton Lukashchuk ${ }^{1} \&$ Tobias J. Kippenberg ${ }^{1}$

Dissipative Kerr solitons (DKS) in optical microresonators provide a highly miniaturised, chipintegrated frequency comb source with unprecedentedly high repetition rates and spectral bandwidth. To date, such frequency comb sources have been successfully applied in the optical telecommunication band for dual-comb spectroscopy, coherent telecommunications, counting of optical frequencies and distance measurements. Yet, the range of applications could be significantly extended by operating in the near-infrared spectral domain, which is a prerequisite for biomedical and Raman imaging applications, and hosts commonly used optical atomic transitions. Here we show the operation of photonic-chip-based soliton Kerr combs driven with 1 micron laser light. By engineering the dispersion properties of a $\mathrm{Si}_{3} \mathrm{~N}_{4}$ microring resonator, octave-spanning soliton Kerr combs extending to $776 \mathrm{~nm}$ are attained, thereby covering the optical biological imaging window. Moreover, we show that soliton states can be generated in normal group-velocity dispersion regions when exploiting mode hybridisation with other mode families.

\footnotetext{
${ }^{1}$ École Polytechnique Fédérale de Lausanne (EPFL), Laboratory of Photonics and Quantum Measurements (LPQM), 1015 Lausanne, Switzerland. Correspondence and requests for materials should be addressed to T.J.K. (email: tobias.kippenberg@epfl.ch)
} 
M icroresonator-based frequency combs are optical frequency combs generated from a continuous-wave (CW) laser via parametric four-wave mixing processes in high- $Q$ microresonators ${ }^{1}$. They have attracted significant interest owing to their compactness, CMOS-compatible fabrication, and ability to operate with repetition rates in the gigahertz to terahertz range with a broad spectral bandwidth ${ }^{2}$. It was recently demonstrated that such $\mathrm{CW}$ laser-driven microresonators can support the spontaneous formation of dissipative Kerr solitons ${ }^{3}$ (DKS)self-organised stable intracavity pulses relying on the double balance between dispersion and nonlinearity ${ }^{4}$, and parametric gain and cavity losses-which provide a route to fully coherent optical frequency combs whose spectral bandwidth can be significantly broadened via soliton-induced Cherenkov radiation ${ }^{5-7}$. Such microresonator-based DKS combs were demonstrated in several platforms $3,5,8,912$ and have already been successfully applied in optical coherent communications ${ }^{10}$, dual-comb spectroscopy ${ }^{11}$, implementation of the microwave-to-optical link via self-referencing 13,14 and most recently in dual-comb distance measurements $^{9,16}$. Yet, to date, well-identified and single DKS have only been attained in the telecommunication bands (around 1550 and $1300 \mathrm{~nm}$ ), where a wide variety of DKS-supporting microresonator platforms have been developed.

Importantly, a large class of new applications of biomedical nature can be accessed with DKS-based comb sources if they can operate in the short-wavelength region of the near-infrared (NIR) domain ranging from 0.7 to $1.4 \mu \mathrm{m}$ wavelength. This spectral region is used for biological and medical imaging due to the highest penetration depth in biological tissues 17 . Optical spectroscopy, Raman spectro-imaging and optical coherence tomography (OCT) techniques operating in this wavelength range serve as a non-invasive tool for the structural and chemical analysis of various biological samples, including retinal and choroidal structures or tumour formations ${ }^{18-20}$. These biomedical imaging techniques could benefit from employing optical frequency combs as light sources due to their coherence and high power per comb line ${ }^{21}$, as well as from using dual-comb approaches allowing to interrogate the broad spectral bands with a single photodetector ${ }^{22}$. A specific example of dual-combbased spectroscopy in the NIR is coherent anti-Stokes Raman spectroscopy (CARS $)^{23}$, which potentially can utilise high repetition rates of the DKS combs for vastly increased acquisition rate, enabling real-time CARS imaging. Furthermore, the luminescence-free anti-Stokes response can benefit from the larger Raman cross section and reduced focal spot size at shorter wavelengths, facilitating phase matching. Such dual-DKS-comb CARS might be able to provide ultrafast multispectral in vivo imaging for chemical, biological and medical purposes. Equally important, a range of other applications requiring a stabilised operation can be accessed and improved by NIR DKS-based combs. The NIR domain hosts optical frequency standards in alkali vapours (e.g. ${ }^{87} \mathrm{Rb},{ }^{133} \mathrm{Cs}$ ), necessary to realise chip-scale optical atomic clocks with enhanced precision ${ }^{24}$, or employed in DKS-comb-based calibration for astronomical spectrometers ${ }^{25}$.

Despite the large number of promising applications of NIR DKS-based combs, such sources with full integration have so far not been developed. Although attempts to generate Kerr combs in the NIR and visible domain have been made ${ }^{26-30}$, they resulted in relatively narrow and incoherent combs, hence soliton formation has not been achieved. The generation of NIR or visible soliton combs is compounded by the increased normal group-velocity dispersion (GVD) of the materials due to the electronic bandgap in UV, and the increased scattering losses and sensitivity to the resonator waveguide dimensions which require higher precision in dispersion engineering and fabrication processes. Moreover, as shown recently, competition between Raman and Kerr effects in the NIR or visible domains can inhibit soliton formation ${ }^{31}$.

In this work, we demonstrate that $\mathrm{Si}_{3} \mathrm{~N}_{4}$ microresonators can overcome these challenges and the DKS-based frequency combs can be generated with a $1060 \mathrm{~nm}$ CW laser, thereby allowing access to the optical wavelength window for biological imaging $(0.7-1.4 \mu \mathrm{m})$. The typical signatures $3,32,33$ of DKS in microresonators, including low-phase-noise operation, Raman-induced red spectral shift, soliton switching dynamics and the bistabilityrelated double-peak response of the microresonator system are observed and provide unambiguous identification of the DKS states. Equally important, we demonstrate the formation of octave-spanning soliton states in this wavelength region by exploiting the coherent dispersive wave emission for efficient spectral broadening towards visible wavelengths. Finally, we report the soliton formation in hybridised microresonator modes, which represents an alternative approach to extend the DKS operation further into the visible domain where normal GVD is usually dominant. The DKS states are achieved in the region of an avoided modal crossing, where the strong interaction between resonator modes leads to locally enhanced anomalous GVD allowing DKS formation. We show that the bandwidth of such soliton states is highly sensitive to the pumped resonance within the interaction region-a behaviour contrasting the behaviour in the absence of modal crossings.

\section{Results}

Device design and characterisation. We employed the silicon nitride $\left(\mathrm{Si}_{3} \mathrm{~N}_{4}\right)$ microresonator platform, which is a welldeveloped and extensively used basis for on-chip nonlinear and quantum photonics due to a number of advantages such as CMOS-compatibility, high effective nonlinearity, negligible twophoton absorption and wide transparency window spanning from visible to mid-infrared region ${ }^{34-36}$. Recent advances in the fabrication processes have enabled the fabrication of crack-free and low-loss $\mathrm{Si}_{3} \mathrm{~N}_{4}$ waveguides with void-free coupling gaps, which guarantee high- $Q$ resonators with well-controllable properties ${ }^{7,37-40}$. An important advantage of this is the ability to engineer the dispersion properties of the microresonators by compensating the material dispersion with the geometry-dependent waveguide dispersion contribution ${ }^{5-7}$. In the context of microresonator-based Kerr frequency combs, the dispersion properties are often expressed through the frequency deviations from an equidistant grid for a certain pumped mode $\omega_{0}$ in the relative mode index $(\mu)$ representation: $D_{\text {int }}(\mu)=\omega_{\mu}-\left(\omega_{0}+\right.$ $\left.D_{1} \mu\right)=\sum_{i>1} D_{i} \mu^{i} / i !, i \in \mathbb{N}$, where $\omega_{\mu}$ is the angular frequency of the cavity resonance, and $\frac{D_{1}}{2 \pi}$ is the free-spectral range (FSR) of a microresonator ${ }^{3}$. For bright DKS generation, it is generally required to achieve anomalous GVD: $D_{2}>0$, which can be especially challenging at short wavelengths.

In this work, we used $\mathrm{Si}_{3} \mathrm{~N}_{4}$ microrings with FSR of $\sim 1 \mathrm{THz}$ (radius $\sim 23 \mu \mathrm{m}$, see Fig. 1b), which were fabricated using the photonic Damascene process ${ }^{38}$. The resonator waveguide width was varied from 1.3 to $1.5 \mu \mathrm{m}$, and the height was targeted at 0.74 $\mu \mathrm{m}$ (with process-related variations in the order of $20 \mathrm{~nm}$ ). The geometry was chosen based on FEM simulations (see Fig. 1c), in order to ensure anomalous GVD of the fundamental TM mode around the pumping wavelength of $1060 \mathrm{~nm}$ (we note that the fundamental TE mode has normal GVD at the same wavelength in all fabricated geometries). The bus waveguide has a pulley-style coupling section with altering width ranging from $0.3 \mu \mathrm{m}$ at the in-coupling part (see Fig. $1 \mathrm{~b}-\mathrm{C}$ ) to $0.65 \mu \mathrm{m}$ at the out-coupling part (see Fig. 1b - A, B). The coupling section was designed to guarantee broadband and good ideality coupling, reaching $\sim 0.85$ at $1 \mu \mathrm{m}$ according to our simulations ${ }^{41}$, which can be further 
a

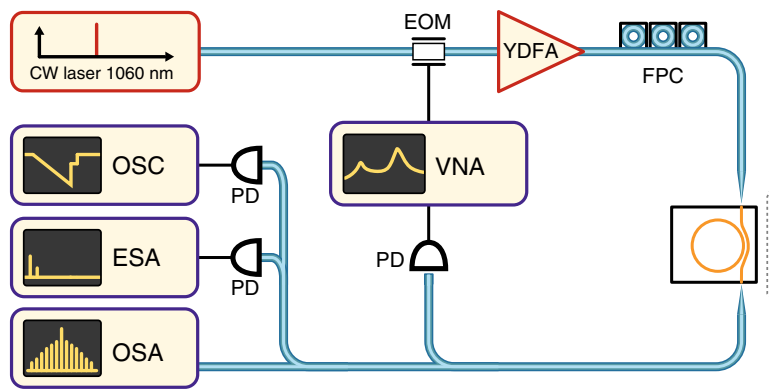

C

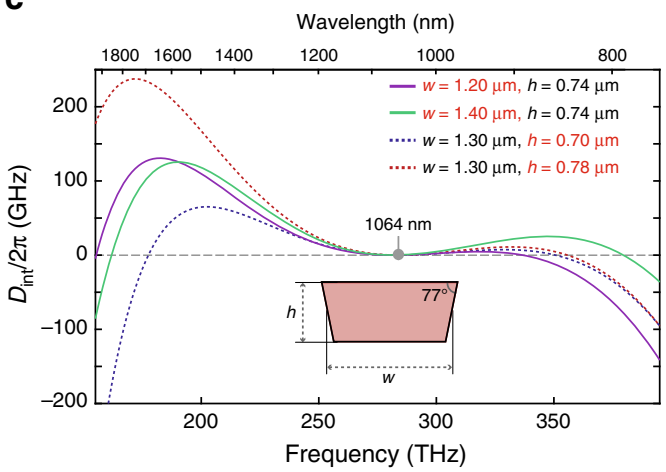

b

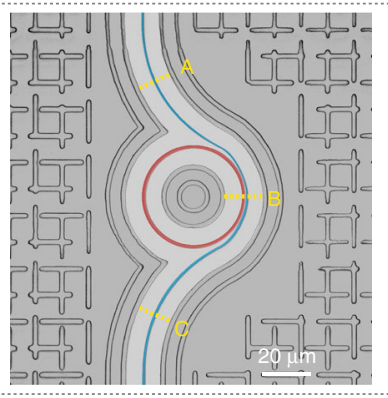

d

Wavelength $(\mathrm{nm})$

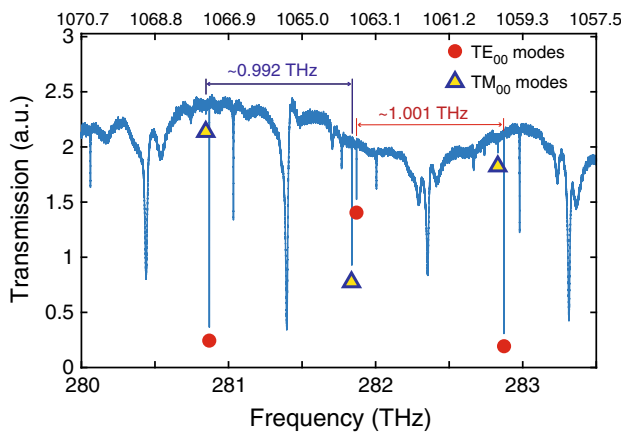

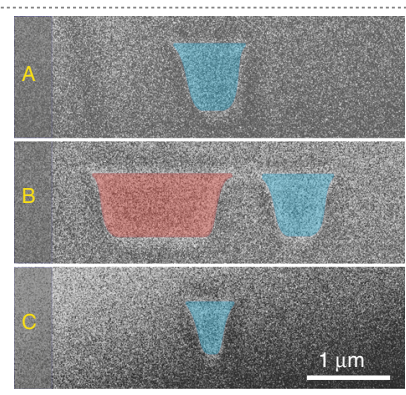

e

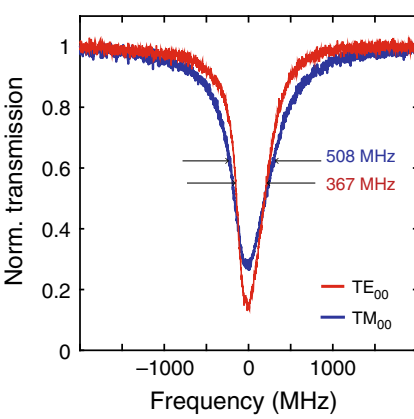

Fig. $1 \mathrm{Si}_{3} \mathrm{~N}_{4}$ microresonators for soliton frequency comb generation in the NIR. a Set-up schematic used for dissipative Kerr soliton generation and characterisation: a tunable external-cavity diode laser with a centre wavelength of $1050 \mathrm{~nm}$ is used as a seed, YDFA - ytterbium-doped fibre amplifier, FPC - fibre polarisation controller, VNA - vector network analyser, EOM - electro-optical phase modulator, PD - photodiode, OSC - oscilloscope, ESA electrical spectrum analyser, OSA - optical spectrum analyser. b Left: optical microscope image of the 1-THz microring resonator (highlighted in red) with a pulley-style bus waveguide (blue). Right: scanning electron microscope images of the resonator and bus waveguide cross sections obtained via focused ion beam cuts at different positions $(A-C)$ marked on the left image. c Simulated integrated dispersion profiles $\left(D_{\text {int }} / 2 \pi\right)$ for TMoo mode of resonator waveguides having different heights of $0.70,0.74$ and $0.78 \mu \mathrm{m}$, widths of 1.2, 1.3 and $1.4 \mu \mathrm{m}$ and fixed sidewall angle of $77^{\circ}$ (see more details on dispersion engineering in Supplementary Note 1). d Transmission trace of the 1-THz microresonator shown in (b). The two fundamental mode families ( $\mathrm{TE}_{00}$ and $\mathrm{TM}_{\mathrm{OO}}$ ) can be distinguished based on their free spectral ranges $\left(0.992 \mathrm{THz}\right.$ for $\mathrm{TM}_{\mathrm{OO}}$ and $1.001 \mathrm{THz}$ for $\mathrm{TE}_{\mathrm{OO}}$ ), and are marked with red and blue shapes correspondingly. Other resonances correspond to higher order modes with comparably lower Q-factors. e Linewidth measurements of the fundamental mode families. The frequency axis was calibrated using a fibre-loop cavity. Typical loaded linewidth of the modes is $\sim 400 \mathrm{MHz}$ for $\mathrm{TE} \mathrm{E}_{00}$ and $\sim 500 \mathrm{MHz}$ for $\mathrm{TM}_{\mathrm{OO}}$

optimised to reduce parasitic losses. The waveguides have inverse tapered mode converters (reducing the waveguide width down to $\sim 150 \mathrm{~nm}$ ) at the input and output ends, providing $<3 \mathrm{~dB}$ coupling loss per facet at $1 \mu \mathrm{m}$.

A typical transmission trace of the fabricated devices is shown in Fig. 1d. The comparably large width of the microring waveguide induces significant overmoding of the resonator. Two fundamental modes- $\mathrm{TE}_{00}, \mathrm{TM}_{00}$ can be easily identified due to different FSRs $\left(0.992 \mathrm{THz}\right.$ for $\mathrm{TM}_{00}$ and $1.001 \mathrm{THz}$ for $\mathrm{TE}_{00}$ ) provided by the non unity aspect ratio. Different coupling of the TE and TM fundamental modes over the measured range in Fig. 1d is attributed to the polarisation plane rotation in the optical fibre before coupling to the on-chip bus waveguide. Once the polarisation is properly adjusted at a certain wavelength for the resonance of a particular mode family, one can measure its $Q$ factor. Both the fundamental modes have comparable loaded linewidths of $370-550 \mathrm{MHz}$, corresponding to the $Q$-factors of $0.55-0.75 \times 10^{6}$ (see Fig. 1e).

Dissipative Kerr solitons at $\mathbf{1} \boldsymbol{\mu m}$. The experimental set-up for the DKS generation in $\mathrm{Si}_{3} \mathrm{~N}_{4}$ microresonators is shown in Fig. 1a. The light from a tunable $1060 \mathrm{~nm}$ CW diode laser (Toptica CTL 1050) is amplified with an ytterbium-doped fibre amplifier and coupled to the chip through a lensed fibre. The output signal was collected with another lensed fibre, and its spectral and noise characteristics were analysed. We also employed a recently developed soliton probing technique ${ }^{33}$, which uses a phasemodulated pump and a vector network analyser to retrieve the system response, allowing us to unambiguously identify DKS formation and track the detuning of the pump from a cavity resonance.

It is well-known, that the CW-driven nonlinear cavity can support DKS states when operating in the effectively red-detuned regime $\left(\omega_{p}-\omega_{0}=2 \pi \delta>0\right.$, where $\omega_{p}$ and $\omega_{0}$ are the angular frequencies of the pump laser and pumped resonance, respectively $)^{3}$. A standard approach for accessing such states is the laser-tuning method ${ }^{3,33}$, where the pump laser is swept over the resonance from the blue to the red side, and is stopped at a certain pump-resonance detuning $\left(2 \pi \delta>\frac{\sqrt{3}}{2} \kappa\right.$, where $\kappa / 2 \pi$ is the total microresonator linewidth) supporting soliton formation. For the samples used in our work, it is sufficient to apply this standard approach, rather than complex techniques such as power kicking ${ }^{5}$ or fast frequency modulation ${ }^{42}$. This can be explained with the relatively small pump powers used for soliton generation. They allowed to avoid the region of transient chaos ${ }^{43}$, causing the elimination of intracavity pulses during the transition to a stable soliton state. In this case, thermal effects associated with the change of intracavity power do not significantly affect the pump-cavity detuning, and the system stays within the soliton existence range after the DKS are formed ${ }^{44}$. 
a
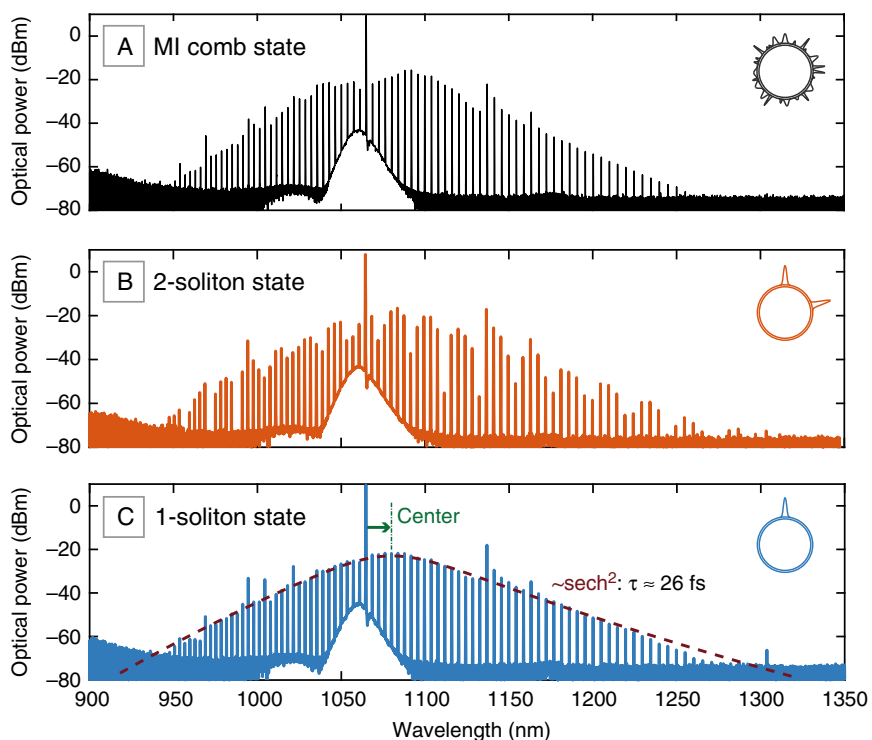

b

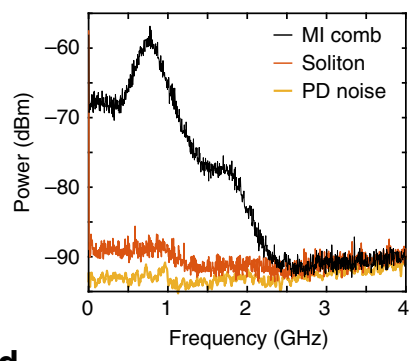

d

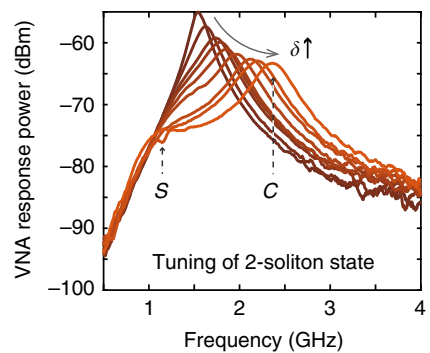

C

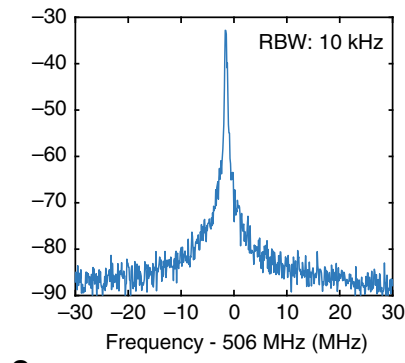

e

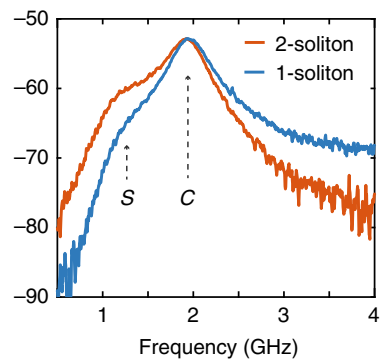

Fig. 2 Dissipative Kerr solitons at $1 \mu \mathrm{m}$ and their characterisation. a Optical spectra of the modulation-instability (MI) comb state (A) and two soliton states ( $B$ and $C$ ) obtained in a 1- $\mathrm{THz} \mathrm{Si}_{3} \mathrm{~N}_{4}$ microresonator (pump is located at around $1065 \mathrm{~nm}$ ). The transition from two-soliton state (B) to single-soliton state (C) was obtained by backward tuning technique of the pump laser. Insets show the estimated positions of the DKS in corresponding states. The singlesoliton state was fitted with the sech ${ }^{2}$ envelope (dashed dark blue) for an estimated duration of $26 \mathrm{fs}$. The green arrow shows the Raman-induced red spectral shift of the soliton spectrum with respect to the pump line. $\mathbf{b}$ Intensity noise of Ml comb state (black), soliton states (red) shown in Fig. $1 \mathbf{a}$ and the noise floor of the photodiode (PD) used for measurements (yellow). c Heterodyne beatnote of the soliton comb line around $1050 \mathrm{~nm}$ with a second CW laser. $\mathbf{d}$ System response evolution in the two-soliton state shown in $\mathbf{a}$, when increasing the pump-cavity detuning $(\delta)$. The positions of characteristic $\mathcal{C}$-resonance and $\mathcal{S}$-resonance are indicated with $C$ and $S$ letters correspondingly. e System response evolution when the DKS state is switched from twosoliton to single-soliton state. The amplitude of the $\mathcal{S}$-resonance has decreased, because the number of intracavity solitons is reduced

We apply this technique with an on-chip pump power of $\sim 1 \mathrm{~W}$ to the resonances of fundamental TM mode family, which should provide anomalous GVD at around $1060 \mathrm{~nm}$. Low tuning speeds on the order of few $\mathrm{GHz} /$ second (i.e., using the laser cavity piezo) were chosen and enabled simultaneous monitoring of the cavity state by measuring the system response signal, as well as an optical spectrum of the output light. The cavity reveals modulation instability and noisy Kerr comb formation while the pump laser is on the blue side of the resonance (see Fig. 2 (a, top)). Upon further pump tuning, the transition to the soliton regime is accompanied by a change of the optical spectrum to a secant hyperbolic-like shape, and the appearance of a doublepeak structure in the system response representing the coexistence of the soliton ( $\mathcal{S}$-resonance) and the CW-background $(\mathcal{C}$-resonance) components inside the cavity. To explore the soliton existence range of the generated DKS state, response measurements were also carried out, while tuning the pump laser towards longer wavelength corresponding to the increase of effective detuning (Fig. 2d). The expected shift in the position of $\boldsymbol{C}$-resonance (which has been shown to indicate the effective detuning of the system) to higher frequencies is clearly seen, and essentially reproduces the dynamics of similar response signals measured for DKS at $1550 \mathrm{~nm}^{33}$. Finally, the transition to the soliton regime has also been verified by the drastic reduction of output-light-intensity-noise (see Fig. 2b), and a narrow heterodyne beatnote of the selected comb line at around $1050 \mathrm{~nm}$ with another CW diode laser (Fig. 2c).

We also demonstrated that the obtained soliton states can experience switching by applying the recently reported backward tuning technique, which relies on the thermal nonlinearity of microresonators, and allows the number of DKS circulating inside the cavity to be changed in a robust and controllable way ${ }^{33}$. Figure 2a shows the switching from a two-soliton state to a single- soliton state. The switching has also been confirmed with the response measurements as shown in Fig. 2e. A decrease in the amplitude of the soliton-number-related $\mathcal{S}$-resonance of the response indicates the reduction in intracavity number of pulses, while the cavity-related $\mathcal{C}$-resonance is almost unchanged. By fitting the spectrum of the final single-soliton state with a sech ${ }^{2}$ envelope, the soliton duration can be estimated from its $3-\mathrm{dB}$ bandwidth as $26 \mathrm{fs}$. We also note the significant soliton red spectral shift $(\sim 4.1 \mathrm{THz}$ in the present case) with respect to the pump line, which is mainly attributed to the Raman effect and observed for all DKS states ${ }^{32,45}$.

Octave-spanning soliton states. Reaching the octave-spanning operation of DKS is an important step in the development of Kerr frequency combs, as it enables the common $f-2 f$ scheme for the offset frequency detection and self-referencing required by multiple applications in optical frequency metrology and low-noise microwave synthesis ${ }^{46}$. Octave-spanning DKS states have only very recently been demonstrated experimentally ${ }^{6,7}$. Here, we demonstrate that DKS-based combs operating at $1 \mu \mathrm{m}$ can also be engineered to have octave-spanning bandwidths, despite operating close to the normal GVD region in the $\mathrm{Si}_{3} \mathrm{~N}_{4}$ platform.

We used a microresonator with the same FSR of $1 \mathrm{THz}$ as in the previous section, having a waveguide geometry of $1.30 \times 0.74$ $\mu \mathrm{m}$, which was designed to maintain the low anomalous GVD and satisfy phase-matching conditions at around $800 \mathrm{~nm}$ for dispersive wave formation ${ }^{5}$. This spectral region is particularly interesting due to the presence of optical frequency standards based on the two-photon $\mathrm{Rb}$ transitions ${ }^{47}$, which can be used for comb referencing. We applied the aforementioned low-speed tuning technique with an estimated on-chip power of $\sim 800 \mathrm{~mW}$ 
a

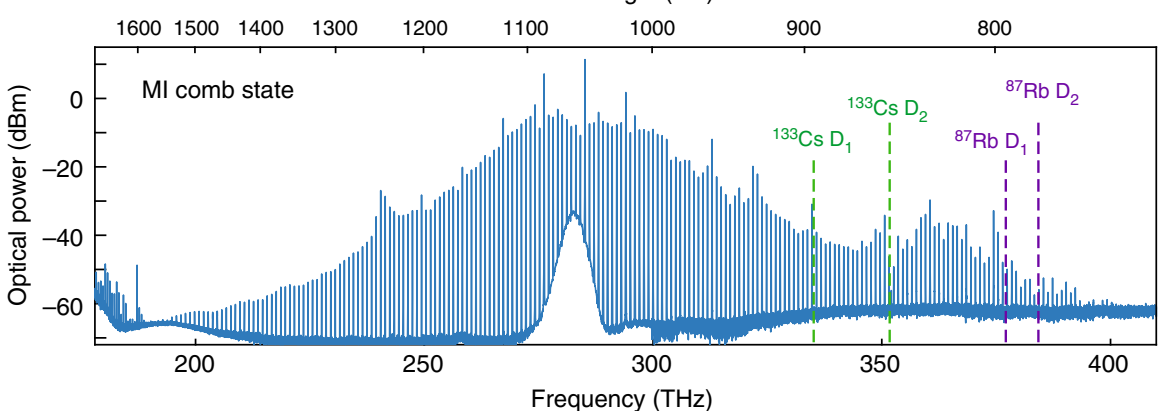

b

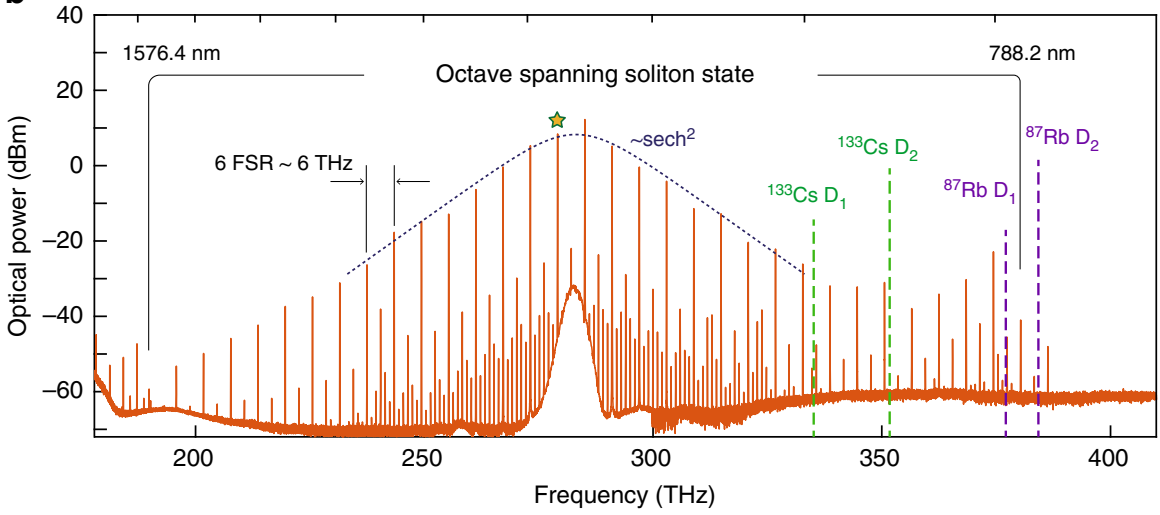

C

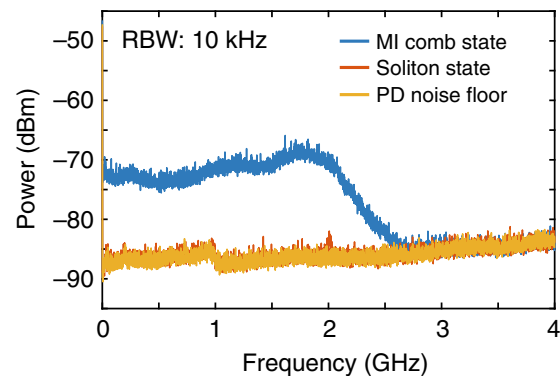

d

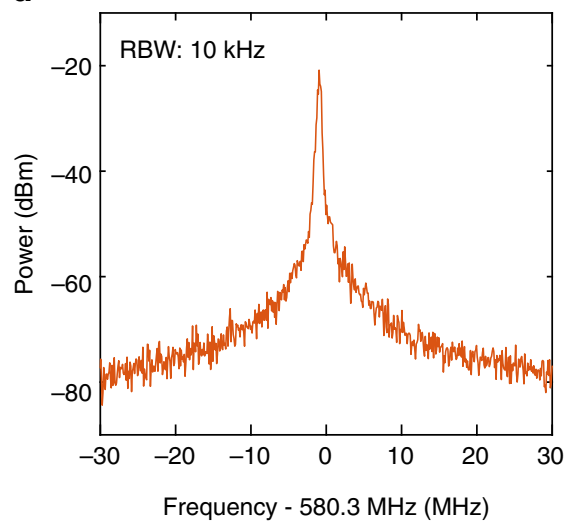

Fig. 3 Octave-spanning microresonator-based dissipative Kerr soliton state in the biological imaging window. a Spectrum of a noisy Kerr comb state obtained with $~ 800 \mathrm{~mW}$ on-chip power (pump is located at around $1051.5 \mathrm{~nm}$ ), from the microresonator with optimised dispersion for octave-spanning operation. Green and purple dashed vertical lines indicate the spectral locations of Cs and Rb optical atomic transitions. $\mathbf{b}$ Spectrum of the octave-spanning soliton state obtained in the same microresonator. The spectrum is fitted with sech ${ }^{2}$ envelope (dashed dark blue), from which a soliton duration of 17 fs is inferred. The set of enhanced lines below $190 \mathrm{THz}$ corresponds to the second diffraction order of optical spectrum analyser diffraction grating, and are thus artefacts. c Intensity noise of the octave-spanning MI comb state (blue) and soliton state (red) shown in (a). Yellow trace shows the noise floor of the photodetector used for measurements. d Heterodyne beatnote of the soliton comb line (marked with a star in panel (b)) with a second CW laser

to achieve the formation of the noisy comb (see Fig. 3a) followed by the soliton state (Fig. 3b).

The resulting spectrum of the soliton state spans over an octave ranging from 776 to $1630 \mathrm{~nm}(>200 \mathrm{THz})$. As expected, it is significantly extended towards shorter wavelengths due to the emission of the dispersive wave via soliton-induced Cherenkov radiation at $800 \mathrm{~nm}^{48}$. The $3-\mathrm{dB}$ bandwidth of the spectrum fitted with the sech $^{2}$ envelope is estimated as $18 \mathrm{THz}$, which corresponds to $\sim 18$-fs pulse. A peculiar shape of the DKS state, consisting of several soliton-like spectra with different FSR is attributed to the formation of a multiple-soliton state represented by an ordered co-propagating DKS ensemble-soliton crystal ${ }^{44,49}$. Such soliton crystals are typically formed in the presence of strong local spectrum deviations caused by intermode interactions among transverse mode families (avoided mode crossings), and in contrast to single-soliton states, they are featuring high conversion efficiency (owing to the high number of intracavity pulses), which in the present case approached $50 \%$.

Similar to the DKS states demonstrated in the previous section, the presented state is also characterised by a low-noise perfomance with a strongly suppressed intensity noise in comparison to the noisy modulation-instability (MI) comb (see Fig. 3c), and a narrow heterodyne beatnote of the generated comb lines with another CW laser (Fig. 3d).

Dissipative Kerr soliton states in hybridised modes. The behaviour of Kerr combs, and in particular DKS states are to a large extent defined by the dispersion properties of the cavity.
One of the key requirements for bright DKS formation is the anomalous GVD of the microresonator, which can be achieved by overcompensating the normal material dispersion with waveguide dispersion contribution. At short wavelengths, however, the increased normal material GVD can represent a significant issue (e.g. for $\mathrm{Si}_{3} \mathrm{~N}_{4}$ in the visible domain), as it can hardly be compensated for the fundamental guided modes, thus hindering the bright DKS formation. Apart from the global dispersion landscape comprised of the material-related and waveguide-related components, the dispersion properties also include spectrally localised dispersion modifications (avoided mode crossings, AMX) that are typically caused by the formation of guided hybridised modes, which can appear due to the interaction of different transverse mode families ${ }^{50}$. Although being spectrally localised, such AMX-s can lead to complex and diverse effects on the dynamics of the DKS states, such as dispersive wave formation, soliton recoil, temporal soliton ordering, appearance of quiet operation points and intermode soliton breathing $3,44,49,51,52$. Moreover, AMX has been reported to allow the formation of mode-locked states consisting of dark pulses in resonators with normal GVD ${ }^{53}$.

In this section, we demonstrate that localised strong anomalous GVD of the hybridised modes around AMX can be directly employed for bright soliton generation irrespective of the global dispersion profile. The effect is schematically explained in Fig. 4a, where the simulated integrated dispersion is plotted (solid lines) for several modes of the $1-\mathrm{THz}$ resonator $(0.74 \times 1.45 \mu \mathrm{m}$, sidewall angle $77^{\circ}$ ). We consider the TE fundamental mode family, which according to our simulations has normal GVD 
a

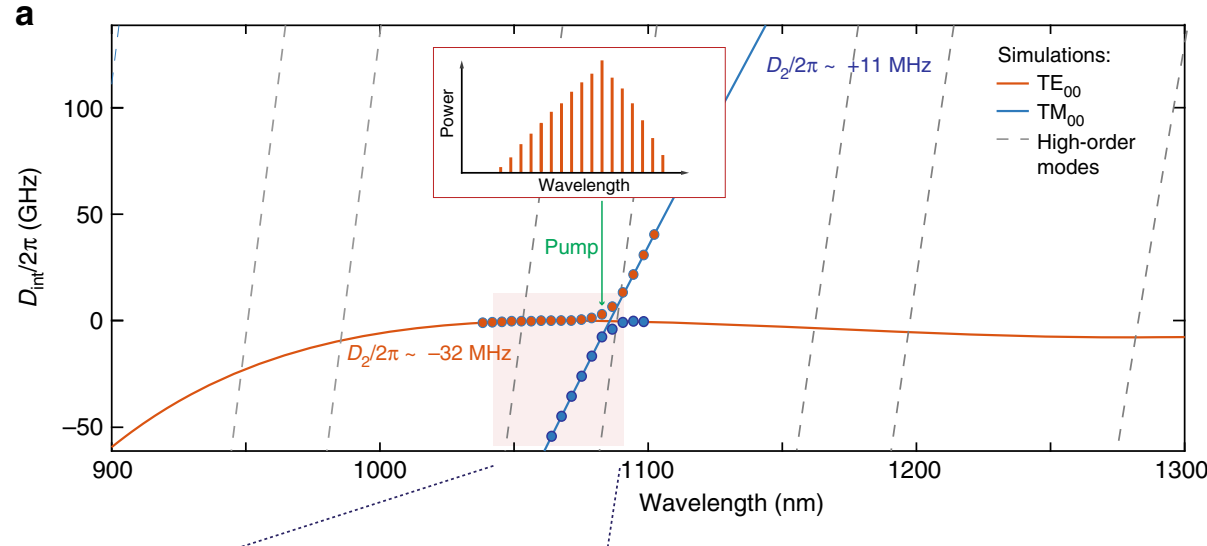

b

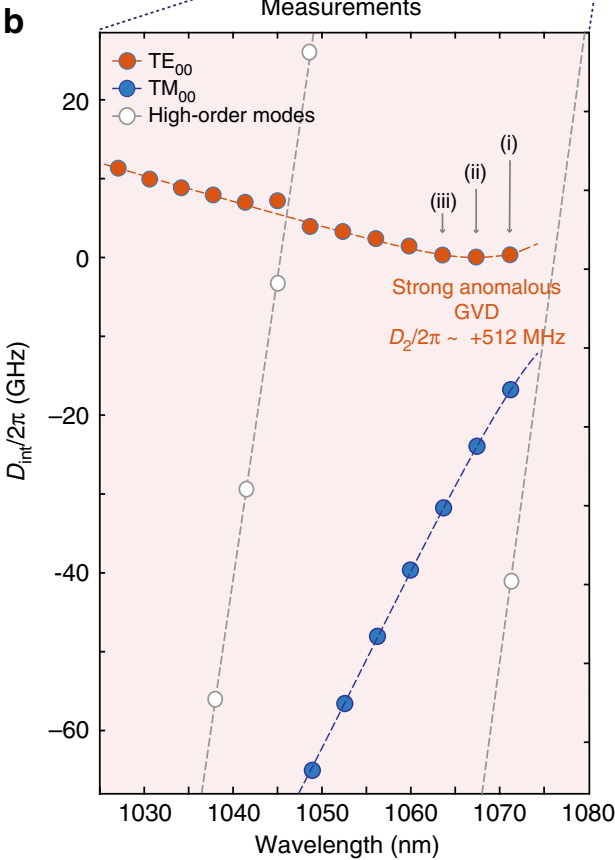

e

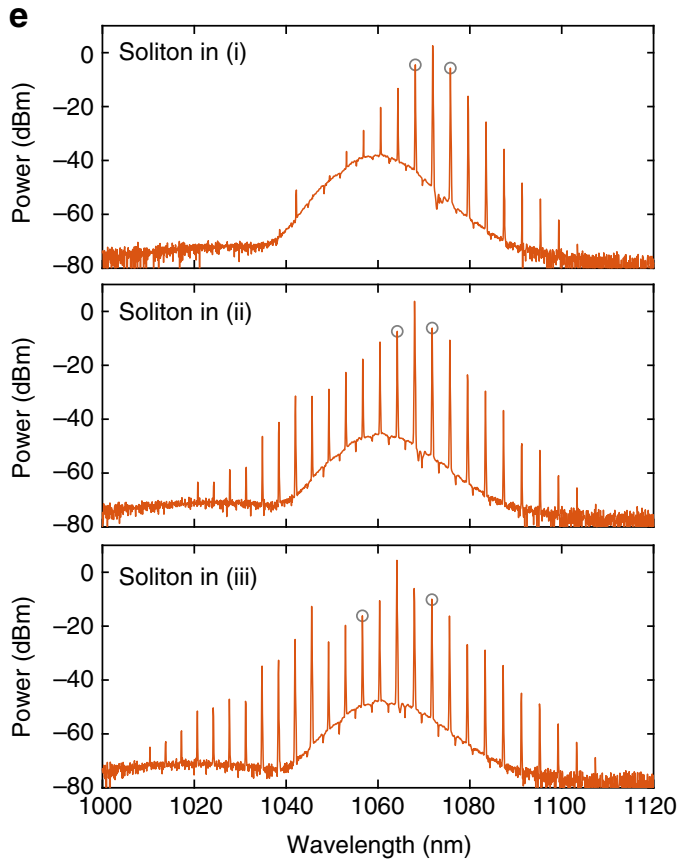

C

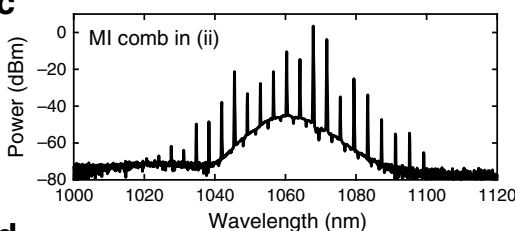

d RBW: $10 \mathrm{kHz}$

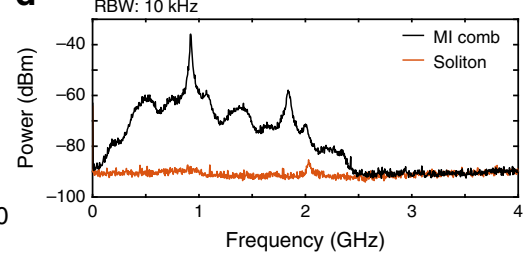

f

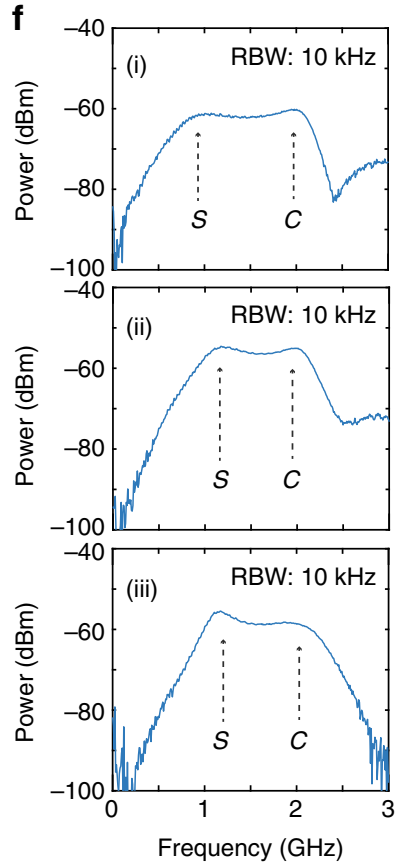

Fig. 4 Dissipative Kerr solitons in hybridised modes. a Scheme of the avoided mode crossing formation. Solid and dashed lines show the simulated integrated dispersion for the fundamental and high-order modes of 1- $\mathrm{THz} \mathrm{Si}_{3} \mathrm{~N}_{4}$ microresonator with dimensions of $1.45 \times 0.74 \mu \mathrm{m}$. Red and blue circles schematically indicate the dispersion profile of the hybridised modes forming AMX. $\mathbf{b}$ Measurements ${ }^{54}$ of the mode structure of a 1-THz $\mathrm{Si}_{3} \mathrm{~N}_{4}$ microresonator with the dimensions of $1.45 \times 0.74 \mu \mathrm{m}$ simulated in (a). Mode families are distinguished based on their FSRs. General dispersion trends cannot be faithfully identified due to the bandwidth limitations of our measurement setup, however a strong local anomalous group-velocity dispersion for three consecutive resonances (i), (ii), (iii) of the TE $E_{00}$ mode family above $1064 \mathrm{~nm}$ can be observed. Dashed lines fit the integrated dispersion of different modes within the measurements range. c Optical spectrum of a noisy comb state, obtained in resonance (ii) from (b). $\mathbf{d}$ Intensity noise measurements of the noisy modulation-instability comb (Ml comb, black) and the dissipative Kerr soliton (soliton, red) states, obtained by pumping the resonance (i) from (b). e Optical spectra of the DKS states obtained by pumping resonances (i), (ii), (iii) from $\mathbf{b}$. Grey circles indicate the positions of primary comb lines. $\mathbf{f}$ Response measurements of DKS states represented in (e). The positions of characteristic $\mathcal{C}$-resonance and $\mathcal{S}$-resonance are indicated with $\mathcal{C}$ and $S$ letters, correspondingly

$\left(\frac{D_{2}}{2 \pi}=-32^{\sim} \mathrm{MHz}\right)$, and note that it has multiple modal crossings with other modes. The frequency degeneracy of different mode families that appears at such modal crossings can lead to mode interaction mediated by scattering processes in the microresonator (see Supplementary Note 3) and induce the formation of AMX. The dispersion profile of one such AMX is schematically shown in Fig. 4a (circles) to highlight its strong deviation from the simulation results obtained in the absence of scattering. Figure $4 \mathrm{~b}$ shows the measured ${ }^{54}$ mode structure of one of the fabricated samples with the same geometry $(0.74 \times 1.45 \mu \mathrm{m})$, where the formation of the described AMX is experimentally observed to be close to the simulated spectral position of $1080 \mathrm{~nm}$ (see Fig. 4b). Mode-interaction-induced strong resonance shifts cause a dramatic change in the local GVD of the TE mode family turning it from normal with $\frac{D_{2}}{2 \pi}=-32 \mathrm{MHz}$ (simulated) to highly anomalous, with $\frac{D_{2}}{2 \pi}$ reaching $510 \mathrm{MHz}$ (measured). We therefore evidence an AMX-induced change in the local GVD.

Driving the modes with such a strong anomalous GVD (when $\sqrt{\frac{\kappa}{D_{2}}}<1$ ) should result in the formation of a natively mode-spaced comb $^{55,56}$, whose primary lines appear 1 FSR away from the pump due to the closely located MI gain peaks. Previous works have reported that such combs can appear directly in a mode-locked regime ${ }^{57}$ which, however, is in contrast with our observations. In the experiments, we again used the same pump tuning technique as 
in previous sections, applied to hybridised modes forming AMX. Using the system response and intensity noise measurements, we observe the standard soft-excitation-route of a Kerr comb formation, which includes the appearance of primary comb lines, development of chaotic modulation instability (MI, Fig. 4c) and the transition to a soliton regime (Fig. 4e), which was verified by the appearance of a characteristic dual-peak system response and lowintensity noise (Fig. 4e,f, see also Supplementary Note 2 for more details). Similar behaviour was observed over three consecutive resonances (cases I, II, III in Fig. 4e), where the corresponding soliton states were generated. An interesting observation can be made regarding the spectral bandwidth of the obtained soliton states. As a result of the localised character of the anomalous GVD of the hybridised modes, the actual value of the dispersion terms (and particularly $\frac{D_{2}}{2 \pi}$ term) varies from one resonance to another, leading to the different effective detunings for generated comb lines and altering the resulting spectral width. We also note here, that due to the contribution of higher-order dispersion terms around AMX, the obtained DKS spectra cannot be faithfully fitted with the $\operatorname{sech}^{2}$ envelope.

The demonstrated soliton states generated in the hybridised modes by exploiting their strong anomalous GVD can represent an alternative way to deterministically generate soliton-based optical combs in arbitrary wavelength regions. This approach can be especially useful for the $\mathrm{Si}_{3} \mathrm{~N}_{4}$ platform presented here to generate soliton states operating at $780 \mathrm{~nm}$ or even further into the visible domain, where normal GVD cannot be efficiently compensated with resonator waveguide geometry, but can be locally altered using e.g., predesigned or thermally controlled $\mathrm{AMXs}^{58}$.

\section{Discussion}

In conclusion, we show the photonic-chip-integrated soliton-based optical frequency comb sources driven with $1 \mu \mathrm{m}$ pump source. The spectra of the demonstrated DKS states are able to span over an octave and cover the common optical frequency standards in alkali vapours, as well as a significant part of the NIR biological imaging window. Moreover, we show that DKS states can be generated in hybridised microresonator modes around avoided mode crossings by directly exploiting their localised anomalous GVD, which represents an alternative approach for the generation of DKS combs in regions with strong normal GVD (e.g. at shorter wavelength in $\mathrm{Si}_{3} \mathrm{~N}_{4}$ and in other materials). From a broader perspective, our work gives strong evidence of the technological readiness of the $\mathrm{Si}_{3} \mathrm{~N}_{4}$ platform for soliton-based operation in the NIR domain around $1 \mu \mathrm{m}$, including comparably good quality factors and the means of dispersion engineering, which makes it a highly promising candidate for multiple biological and other applications in this spectral window, including OCT and dualcomb CARS. Finally, we would like to draw the reader's attention to the other work on the generation of DKS states centred at 1064 and $780 \mathrm{~nm}$ in fibre-coupled silica microdiscs[59].

Data availability. The code and data used to produce the plots within this paper are available at 10.5281/zenodo.1149180. All other data used in this study are available from the corresponding authors upon reasonable request.

Received: 26 June 2017 Accepted: 16 February 2018

Published online: 20 March 2018

\section{References}

1. Del'Haye, P. et al. Optical frequency comb generation from a monolithic microresonator. Nature 450, 1214-1217 (2007).
2. Kippenberg, T. J., Holzwarth, R. \& Diddams, S. Microresonator-based optical frequency combs. Science 332, 555-559 (2011).

3. Herr, T. et al. Temporal solitons in optical microresonators. Nat. Photonics $\mathbf{8}$, 145-152 (2014).

4. Akhmediev, N. N. \& Ankiewicz, A. Solitons Around Us: Integrable, Hamiltonian and Dissipative Systems, in Optical Solitons, pp. 105-126. (Springer, Berlin, Heidelberg, 2002)

5. Brasch, V. et al. Photonic chip-based optical frequency comb using soliton Cherenkov radiation. Science 351, 357-360 (2016).

6. Li, Q. et al. Stably accessing octave-spanning microresonator frequency combs in the soliton regime. Optica 4, 193-203 (2017).

7. Pfeiffer, M. H. et al. Octave-spanning dissipative Kerr soliton frequency combs in $\mathrm{Si}_{3} \mathrm{~N}_{4}$ microresonators. Optica 4, 684-691 (2017).

8. Yi, X., Yang, Q.-F., Yang, K. Y., Suh, M.-G. \& Vahala, K. Soliton frequency comb at microwave rates in a high-Q silica microresonator. Optica 2, 1078-1085 (2015)

9. Webb, K. E., Erkintalo, M., Coen, S. \& Murdoch, S. G. Experimental observation of coherent cavity soliton frequency combs in silica microspheres. Opt. Lett. 41, 4613-4616 (2016).

10. Marin-Palomo, P. et al. Microresonator-based solitons for massively parallel coherent optical communications. Nature 546, 274-279 (2017).

11. Suh, M.-G., Yang, Q.-F., Yang, K. Y., Yi, X. \& Vahala, K. J. Microresonator soliton dual-comb spectroscopy. Science 354, 600-603 (2016).

12. Yu, M., Okawachi, Y., Griffith, A. G., Lipson, M. \& Gaeta, A. L. Mode-locked mid-infrared frequency combs in a silicon microresonator. Optica 3, 854-860 (2016).

13. Brasch, V., Lucas, E., Jost, J. D., Geiselmann, M. \& Kippenberg, T. J. Selfreferenced photonic chip soliton Kerr frequency comb. Light: Sci. \& Appl. 6, e16202 (2017)

14. Jost, J. D. et al. Counting the cycles of light using a self-referenced optical microresonator. Optica 2, 706-711 (2015).

15. Trocha, P. et al. Ultrafast optical ranging using microresonator soliton frequency combs. Science 359, 887-891 (2018).

16. Suh, M.-G. \& Vahala, K. Soliton microcomb range measurement. Science 359, 884-887 (2018).

17. Smith, A. M., Mancini, M. C. \& Nie, S. Second window for in vivo imaging Nat. Nanotechnol. 4, 710-711 (2009).

18. Drexler, W. et al. Ultrahigh-resolution ophthalmic optical coherence tomography. Nat. Med. 7, 502-507 (2001).

19. Reich, G. Near-infrared spectroscopy and imaging: basic principles and pharmaceutical applications. Adv. Drug Deliv. Rev. 57, 1109-1143 (2005).

20. Richards-Kortum, R. \& Sevick-Muraca, E. Quantitative optical spectroscopy for tissue diagnosis. Annu. Rev. Phys. Chem. 47, 555-606 (1996).

21. Siddiqui, M., Vakoc, B. J., Nam, A. \& Lippok, N. High-speed subsampled optical coherence tomography imaging with frequency comb lasers. In Conference on Lasers and Electro-Optics, OSA Technical Digest (online), AW4A.2 (Optical Society of America, 2017).

22. Coddington, I., Newbury, N. \& Swann, W. Dual-comb spectroscopy. Optica 3, 414-426 (2016).

23. Ideguchi, T. et al. Coherent Raman spectro-imaging with laser frequency combs. Nature 502, 355-358 (2013).

24. Papp, S. et al. Microresonator frequency comb optical clock. Optica 1, 10-14 (2014).

25. Obrzud, E., et al. A Microphotonic Astrocomb. Preprint at https://arxiv.org/ abs/1712.09526 (2017).

26. Saha, K. et al. Broadband parametric fuency comb generation with a $1-\mathrm{m}$ pump source. Opt. Express 20, 26935-26941 (2012).

27. Guo, X. et al. Efficient visible frequency comb generation via Cherenkov radiation from a Kerr microcomb. Preprint at https://arxiv.org/abs/ 1704.04264 (2017).

28. Wang, L. et al. Frequency comb generation in the green using silicon nitride microresonators. Laser \& Photonics Rev. 10, 631-638 (2016).

29. Savchenkov, A. et al. Kerr combs with selectable central frequency. Nat. Photonics 5, 293-296 (2011).

30. Yang, Y. et al. Four-wave mixing parametric oscillation and frequency comb generation at visible wavelengths in a silica microbubble resonator. Opt. Lett. 41, 5266-5269 (2016).

31. Okawachi, Y. et al. Competition between Raman and Kerr effects in microresonator comb generation. Opt. Lett. 42, 2786-2789 (2017).

32. Karpov, M. et al. Raman self-frequency shift of dissipative Kerr solitons in an optical microresonator. Phys. Rev. Lett. 116, 103902 (2016).

33. Guo, H. et al. Universal dynamics and deterministic switching of dissipative Kerr solitons in optical microresonators. Nat. Phys. 13, 94-102 (2017).

34. Levy, J. et al. CMOS-compatible multiple-wavelength oscillator for on-chip optical interconnects. Nat. Photonics 4, 37-40 (2010).

35. Li, Q., Davanço, M. \& Srinivasan, K. Efficient and low-noise single-photonlevel frequency conversion interfaces using silicon nanophotonics. Nat. Photonics 10, 406-414 (2016). 
36. Moss, D., Morandotti, R., Gaeta, L. \& Lipson, M. New CMOS-compatible platforms based on silicon nitride and hydex for nonlinear optics. Nat. Photonics 7, 597-607 (2013).

37. Gondarenko, A., Levy, J. S. \& Lipson, M. High confinement micron-scale silicon nitride high-Q ring resonator. Opt. Express 17, 11366-11370 (2009).

38. Pfeiffer, M. H. P. et al. Photonic Damascene process for integrated high-Q microresonator based nonlinear photonics. Optica 3, 20-25 (2016).

39. Herkommer, C. et al. Mid-infrared dispersive wave generation in silicon nitride nano-photonic waveguides. Preprint at https://arxiv.org/abs/ 1704.02478 (2017).

40. Kordts, A., Pfeiffer, M. H. P., Guo, H., Brasch, V. \& Kippenberg, T. J. Higher order mode suppression in high-Q anomalous dispersion $\mathrm{SiN}$ microresonators for temporal dissipative Kerr soliton formation. Opt. Lett. 41, 452-455 (2016).

41. Pfeiffer, M. H., Liu, J., Geiselmann, M. \& Kippenberg, T. J. Coupling ideality of integrated planar high-Q microresonators. Phys. Rev. Appl. 7, 024026 (2017).

42. Stone, J. R. et al. Initiating Kerr-soliton frequency combs apart from thermal bistability and mode perturbation effects. In Conference on Lasers and ElectroOptics, OSA Technical Digest (online), STu4J.4 (Optical Society of America, 2017).

43. Leo, F. et al. Dynamics of one-dimensional Kerr cavity solitons. Opt. Express 21, 9180-9191 (2013).

44. Karpov, M. et al. Dynamics of soliton crystals in optical microresonators. In Conference on Lasers and Electro-Optics, OSA Technical Digest (online), FTu1D.2 (Optical Society of America, 2017).

45. Milián, C., Gorbach, A. V., Taki, M., Yulin, A. V. \& Skryabin, D. V. Solitons and frequency combs in silica microring resonators: interplay of the Raman and higher-order dispersion effects. Phys. Rev. A 92, 033851 (2015).

46. Newbury, N. R. Searching for applications with a fine-tooth comb. Nat Photonics 5, 186-188 (2011).

47. Jones, D. J. et al. Carrier-envelope phase control of femtosecond mode-locked lasers and direct optical frequency synthesis. Science 288, 635-639 (2000).

48. Akhmediev, N. \& Karlsson, M. Cherenkov radiation emitted by solitons in optical fibers. Phys. Rev. A 51, 2602-2607 (1995).

49. Cole, D. C., Lamb, E. S., Del'Haye, P., Diddams, S. A. \& Papp, S. B. Soliton crystals in Kerr resonators. Nat. Photonics 11, 671-677 (2016).

50. Savchenkov, A. et al. Kerr frequency comb generation in overmoded resonators. Opt. Express 20, 27290-27298 (2012).

51. Guo, H. et al Intermode breather solitons in optical microresonators. Phys. Rev. X 7, 041055 (2017).

52. Yi, X. et al. Single-mode dispersive waves and soliton microcomb dynamics. Nat. Commun. 8, 14869 (2017).

53. Xue, X. et al. Mode-locked dark pulse Kerr combs in normal-dispersion microresonators. Nat. Photonics 9, 594-600 (2015).

54. Del'Haye, P., Arcizet, O., Gorodetsky, M. L., Holzwarth, R. \& Kippenberg, T. J. Frequency comb assisted diode laser spectroscopy for measurement of microcavity dispersion. Nat. Photonics 3, 529-533 (2009).

55. Ferdous, F. et al. Spectral line-by-line pulse shaping of on-chip microresonator frequency combs. Nat. Photonics 5, 770-776 (2011).

56. Herr, T. et al. Universal formation dynamics and noise of Kerr-frequency combs in microresonators. Nat. Photonics 6, 480-487 (2012).

57. Liu, Y. et al. Investigation of mode coupling in normal-dispersion silicon nitride microresonators for Kerr frequency comb generation. Optica 1, 137-144 (2014).
58. Xue, X. et al. Normal-dispersion microcombs enabled by controllable mode interactions. Laser \& Photonics Rev. 9, L23-L28 (2015).

59. Lee, S. H. et al. Towards visible soliton microcomb generation. Nat. Commun. 8, 1295 (2017).

\section{Acknowledgements}

This publication was supported by Contract W31P4Q-14-C-0050 from the Defence Advanced Research Projects Agency (DARPA), Defence Sciences Office (DSO); by the Air Force Office of Scientific Research, Air Force Material Command, USAF under Award No. FA9550-15-1-0099; We also acknowledge the support from the European Space Technology Centre with ESA Contract No. 4000116145/16/NL/MH/GM and the support from the European Union's FP7 programme under Marie Sklodowska-Curie Initial Training Network grant agreement No. 607493. $\mathrm{Si}_{3} \mathrm{~N}_{4}$ samples were fabricated and grown in the Center of MicroNanoTechnology (CMi) at EPFL.

\section{Author contributions}

M.K. designed and performed experiments and analysed the data. M.P. designed the samples and performed dispersion simulations. A.L. assisted with dispersion simulations. J.L. implemented ideality simulations. M.K. wrote the manuscript with an input from M. P., J.L., A.L., T.J.K. T.J.K. supervised the project.

\section{Additional information}

Supplementary Information accompanies this paper at https://doi.org/10.1038/s41467018-03471-x.

Competing interests: The authors declare no competing interests.

Reprints and permission information is available online at http://npg.nature.com/ reprintsandpermissions/

Publisher's note: Springer Nature remains neutral with regard to jurisdictional claims in published maps and institutional affiliations.

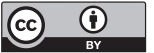

Open Access This article is licensed under a Creative Commons Attribution 4.0 International License, which permits use, sharing, adaptation, distribution and reproduction in any medium or format, as long as you give appropriate credit to the original author(s) and the source, provide a link to the Creative Commons license, and indicate if changes were made. The images or other third party material in this article are included in the article's Creative Commons license, unless indicated otherwise in a credit line to the material. If material is not included in the article's Creative Commons license and your intended use is not permitted by statutory regulation or exceeds the permitted use, you will need to obtain permission directly from the copyright holder. To view a copy of this license, visit http://creativecommons.org/ licenses/by/4.0/.

(C) The Author(s) 2018 